\title{
Impact of Multivessel Coronary Artery Disease on Long Term Prognosis in Patients with ST- segment Elevation Myocardial Infarction
}

\author{
Lidija Savic $^{1,2}$, Igor Mrdovic ${ }^{1,2}$, Milika Asanin ${ }^{1,2}$, Sanja Stankovic ${ }^{3}$, Gordana Krljanac ${ }^{1,2}$, \\ Ratko Lasica ${ }^{1}$ \\ ${ }^{1}$ Coronary Care Unit, Emergency Hospital, Clinical Centre of Serbia, Belgrade, Serbia \\ ${ }^{2}$ Cardiology Clinic, Emergency Hospital, Clinical Centre of Serbia, Belgrade, Serbia \\ ${ }^{3}$ Center for Medical Biochemistry, Emergency Hospital, Clinical Centre of Serbia, Belgrade, Serbia
}

\section{ABSTRACT}

Background: A significant proportion of patients with ST-segment elevation myocardial infarction (STEMI) have multivessel coronary artery disease (MVD), and they are at high risk for recurrent cardiac events. The aim of the present study was to analyze the impact of MVD on long-term cardiovascular mortality in STEMI patients treated with primary percutaneous coronary intervention (pPCI). Method: This study included 3,115 consecutive STEMI patients hospitalized in the Coronary Care Unit of the Clinical Centre of Serbia, between November 2005 and January 2012. Patients were divided in two groups: MVD and no MVD. MVD disease was defined as stenosis greater than $50 \%$ by visual assessment in more than one major coronary artery. Primary PCI was limited to the infarct-related artery (IRA). Cardiovascular mortality was defined as any death from cardiovascular reason (myocardial reinfarction, low-output heart failure, and sudden death). Patients presenting with cardiogenic shock were excluded. Patients were followed-up for 6 years after enrollment. Results: Among 3,115 analyzed patients, 1,352 (43.4\%) patients had no MVD and 1,763 (56.6\%) had MVD; among patients with MVD, 926 (52.6\%) had two-vessel disease and 837 (47.4\%) had three-vessel disease. Compared with patients with single-vessel disease, patients with MVD were older, had longer pain duration, and presented more often with heart failure; they were more likely to have previous coronary artery disease, diabetes, hypertension, and chronic kidney disease; post-procedural flow TIMI <3 was more frequently observed in patients with MVD than in patients with no MVD (5.9\% vs. 3.1\%, p <0.001). Patients with MVD had lower left ventricular ejection fraction than patients with single-vessel disease: $45 \%$ (interquartile range [IQR] 40-55\%) vs. 50\% (IQR 43-55\%), p <0.001. Revascularization of non-IRA lesions was performed at index hospitalization in 1,075 (61\%) patients, and in 602 (34.1\%) patients revascularization was performed in the first few months after PPCI (median 1.5 months, IQR 1-2.5 months); coronary artery bypass grafting was performed in 291 (18.4\%) patients and PCI (with stent implantation) in 1,368 (81.6\%) patients. Six-year cardiovascular mortality was significantly higher in patients with MVD than in patients with single-vessel disease $(10.4 \%$ vs. $4.6 \%, \mathrm{p}<0.001)$. In multivariate Cox regression analysis, MVD remained an independent

\section{ARTICLE HISTORY}

Received: March 26, 2019

Accepted: May 14, 2019

\section{CORRESPONDENCE}

\section{Lidija Savic}

Pasterova 2

11000 Beograd, Serbia

Tel: +38163343276

E-mail: lidijasavic2007@gmail.com 
predictor for 6-year cardiovascular mortality (HR 1.55, 95\% CI 1.11-2.06, p = 0.041). Conclusion: In STEMI patients treated with pPCI, the presence of MVD remained an independent predictor for higher long-term cardiovascular mortality despite early revascularization of the remaining stenosis in non-IRA.

Keywords: pericoronary fat, plaque geometry, CCTA, acute chest pain, vulnerable coronary lesions

\section{INTRODUCTION}

Primary percutaneous coronary intervention (pPCI) is the reperfusion of choice for the treatment of patients with ST-segment elevation myocardial infarction (STEMI). Primary PCI improves survival compared with thrombolysis, mainly due to the large percentage of restoration of TIMI 3 flow through the infarct-related artery (IRA). ${ }^{1-4}$ Concomitant atherosclerosis in coronary vessels other than the IRA is observed in a significant number of STEMI patients..$^{1,2,4^{-9}}$ According to the current guidelines, the standard therapeutic approach in STEMI patients with multi-vessel coronary artery disease (MVD) is represented by pPCI of the IRA in acute settings, followed by the subsequent revascularization of other lesions during the same hospitalization or, rarely, within the first month following discharge. ${ }^{8,10} \mathrm{~A}$ small number of patients are treated conservatively (medically), unless recurrent ischemia occurs. ${ }^{3}$ Many studies so far have shown worse outcomes for STEMI patients with MVD compared to STEMI patients with single-vessel coronary artery disease (SVD) in shortand medium-term follow-up, regardless of the reperfusion strategy. ${ }^{1,3,4-6}$ In STEMI subjects with MVD who undergo interventional therapies with angioplasty, a worse reperfusion rate is also observed when compared with patients with SVD. ${ }^{2,4,6}$ The mechanism through which MVD adversely affects the outcome of patients with STEMI is multifactorial and relatively poorly characterized. ${ }^{1,6}$ To our best knowledge, the longer term impact of MVD on patient outcomes for STEMI treated with contemporary pPCI has not been extensively investigated so far.

The aim of the present study is to analyze the impact of MVD on long-term cardiovascular mortality in STEMI patients treated with pPCI.

\section{METHODS}

The present study used data from the prospective Clinical Centre of Serbia STEMI Registry, for a subgroup of 3,115 consecutive patients who were hospitalized between November 2005 and January 2012. The purpose of the prospective Clinical Centre of Serbia STEMI Registry has been published elsewhere. ${ }^{11}$ In brief, the objective of the registry is to gather complete and representative data on the management and short- and long-term outcomes of patients with STEMI who have undergone primary PCI at the Centre. All consecutive patients with STEMI, aged $>18$ years, who had been admitted to the Coronary Care Unit after undergoing PPCI at the Centre, were included in the Registry. For this study, patients with cardiogenic shock at admission were excluded.

The study protocol was approved by the local research ethics committee. The research was performed in accordance with the code of ethics of the World Medical Association's Declaration of Helsinki. All patients signed a written informed consent before being enrolled in the study.

Coronary angiography was performed via the femoral approach. Aspirin, $300 \mathrm{mg}$, and clopidogrel, $600 \mathrm{mg}$, were administered to all eligible patients before pPCI. Selected patients, with visible intracoronary thrombi, were also given the glycoprotein (GP) IIb/IIIa receptor inhibitor tirofiban during pPCI. Flow grades were assessed according to the Thrombolysis in Myocardial Infarction (TIMI) criteria. Primary PCI was limited to the culprit lesion (IRA) in acute settings. After pPCI, patients were treated according to current guidelines.

The extent of coronary artery disease (CAD) was graded as no MVD and MVD (bi-vascular of three-vascular atherosclerotic involvement). MVD was considered in case of a vascular stenosis of more than $50 \%$, as assessed by visual analysis of the coronary angiography, in more than a single coronary artery. The indication for revascularization (PCI or aorto-coronary bypass grafting, CABG) of additional lesions was decided by the clinician assigned to the case, in collaboration with the interventional cardiology physician. Reperfusion therapy for associated atherosclerotic lesions was achieved during the index hospitalization period, or during the second follow-up.

Demographic, baseline clinical, angiographic, and procedural data were collected and analyzed. Baseline renal dysfunction was defined as an estimated glomerular filtration rate (eGFR) $<60 \mathrm{~mL} / \mathrm{min} / 1.73 \mathrm{~m}^{2}$ at admission (before pPCI). The eGFR was calculated using the Modification of Diet in Renal Disease (MDRD) equation. Echo- 
cardiographic examination was performed within the first 3 days following pPCI. Left ventricular ejection fraction (LVEF) was assessed according to the biplane Simpson method, in classical two- and four-chamber apical projections.

Patients were followed-up for 6 years after enrollment. Follow-up data were obtained by scheduled telephone interviews and out-patient visits. Cardiovascular death included any death due to cardiac cause (myocardial reinfarction, low-output heart failure, fatal arrhythmia) and sudden death.

\section{STATISTICAL ANALYSIS}

Continuous variables were expressed as the median (med), with the interquartile range (IQR) between the 25th and 75th quartiles, whereas categorical variables were expressed as frequency and percentage. Analysis for normality of data (continuous variables) was performed using the Kolmogorov-Smirnov test. Baseline differences between groups were analyzed using the Mann-Whitney test for continuous variables and the Pearson $\chi^{2}$ test for categorical variables. The Kaplan-Meier method was used for constructing probability curves for 6-year cardiovascular mortality, whereas the difference between the groups was tested with the Log Rank test. Multiple logistic regression analysis was used for identifying predictors

TABLE 1. Baseline characteristics and therapy at discharge of the analyzed patients according to presence of MVD

\begin{tabular}{|c|c|c|c|}
\hline Characteristics & $\begin{array}{c}\text { No MVD } \\
N=1,352\end{array}$ & $\begin{array}{c}\text { MVD } \\
\mathrm{N}=\mathbf{1 , 7 6 3}\end{array}$ & $\mathrm{p}$ value \\
\hline Age, years med (IQR) & $56(49-75)$ & $62(55-72)$ & $<0.001$ \\
\hline Male, n (\%) & $974(72)$ & $1,264(71.6)$ & 0.409 \\
\hline Previous MI, n (\%) & $80(5.9)$ & $247(14)$ & $<0.001$ \\
\hline Previous CABG, n (\%) & $17(1.2)$ & $30(1.7)$ & 0.116 \\
\hline Previous PCI, n (\%) & $20(1.5)$ & $30(1.7)$ & 0.235 \\
\hline Diabetes, n (\%) & $197(14.6)$ & $413(23.4)$ & $<0.001$ \\
\hline Hypertension, n (\%) & $814(60.2)$ & $1,276(72.4)$ & $<0.001$ \\
\hline HLP, n (\%) & $804(59.5)$ & $1,086(61.6)$ & 0.229 \\
\hline Smoking, n (\%) & $807(59.7)$ & $852(48.4)$ & $<0.001$ \\
\hline Pain duration, hours med (IQR) & $2(1.5-4)$ & $3(2-5)$ & $<0.001$ \\
\hline $\mathrm{HF}$ at admission, $\mathrm{n}(\%)$ & $117(8.7)$ & $274(15.5)$ & $<0.001$ \\
\hline Systolic BP (mmHg) at admission, med (IQR) & $135(120-150)$ & $140(120-150)$ & 0.081 \\
\hline HR at admission, med (IQR) & $78(70-80)$ & $80(70-90)$ & 0.320 \\
\hline Anterior infarction, n (\%) & $566(41.8)$ & $691(39.2)$ & 0.409 \\
\hline Preprocedural flow TIMI o, n (\%) & $928(68.7)$ & $1,297(73.5)$ & 0.309 \\
\hline Postprocedural flow TIMI <3, n (\%) & $42(3.1)$ & $104(5.9)$ & $<0.001$ \\
\hline IIb/IIIa inhibitors, n (\%) & $495(36.6)$ & $665(37.6)$ & 0.750 \\
\hline LVEF \%, med (IQR) & $50(43-55)$ & $45(40-50)$ & $<0.001$ \\
\hline CKmax, med (IQR) & $1,868(921-3,568)$ & $1,892(967-3,460)$ & 0.268 \\
\hline Troponin I ( $\mu \mathrm{g} / \mathrm{L})$, med (IQR) & $30.77(8.05-79)$ & $31.26(9.71-94.76)$ & 0.169 \\
\hline $\mathrm{eGFR}<60 \mathrm{~mL} / \mathrm{min} / \mathrm{m}^{2}$ & $143(10.6)$ & $346(19.6)$ & $<0.001$ \\
\hline In-hospital death, $\mathrm{n}(\%)$ & $30(2.2)$ & $101(5.8)$ & $<0.001$ \\
\hline \multicolumn{4}{|l|}{ Therapy at discharge* } \\
\hline Beta blockers, n (\%) & $1,164(86.1)$ & $1,415(80.3)$ & 0.757 \\
\hline ACE inhibitors, $\mathrm{n}(\%)$ & $1,074(79.4)$ & $1,330(75.4)$ & 0.230 \\
\hline Statins, n (\%) & $1,155(85.4)$ & $1,399(79.4)$ & 0.636 \\
\hline Diuretics, n (\%) & $165(12.2)$ & $313(17.8)$ & $<0.001$ \\
\hline Nitrates, n (\%) & $125(9.2)$ & $335(19)$ & $<0.001$ \\
\hline Antiarrhythmics, n (\%) & $42(3.1)$ & $40(2.3)$ & 0.229 \\
\hline Ca antagonist, n (\%) & $45(3.4)$ & $60(3.4)$ & 0.649 \\
\hline
\end{tabular}

*all patients had aspirin and clopidogrel at discharge; MVD - multivessel disease; MI - myocardial infarction; CABG - aortocoronary bypass grafting; HLP - hyperlipidemia; HF - heart failure; BP - arterial blood pressure; HR - heart rate; CK - creatinine kinase; eGFR - estimated glomerular filtration rate; LVEF - left ventricular ejection fraction; ACE - angiotensin converting enzyme 


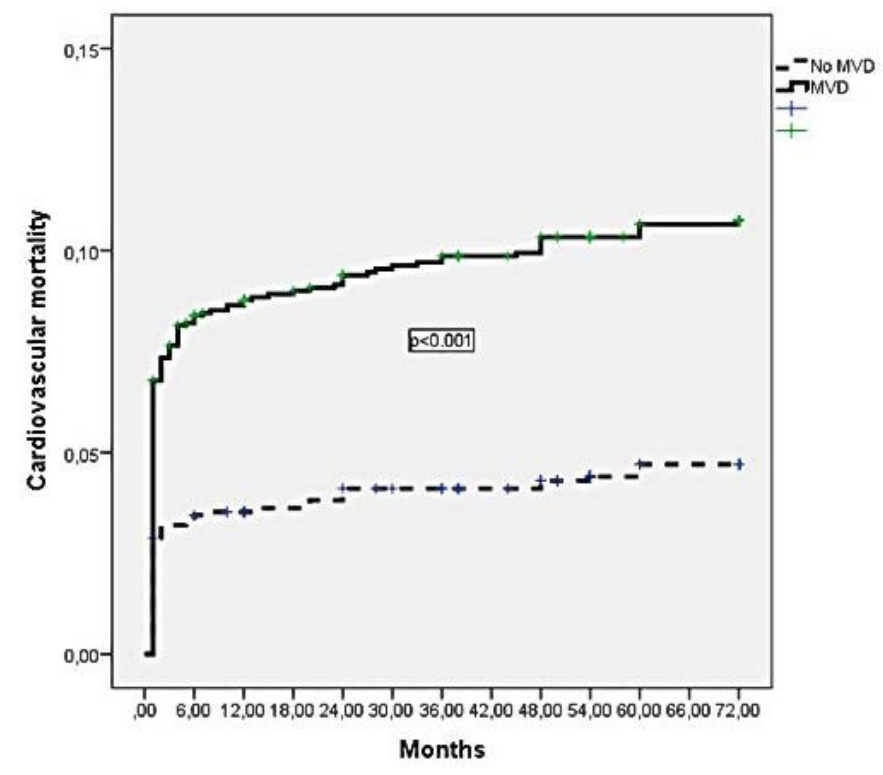

FIGURE 1. Kaplan-Meier curves estimating probability for six-year cardiovascular mortality in patients with MVD and no MVD

for cardiovascular mortality. Multivariate Cox regression analysis (backward method, with $\mathrm{p}<0.10$ for entrance into the model) was used for identifying independent risk factors for the occurrence of 6-year cardiovascular mortality. SPSS statistical software, version 19.0, was applied (SPSS Inc, Chicago, IL).

\section{RESULTS}

The average age of all analyzed patients was 59 (52-69) years. From the total of 3,115 analyzed patients, 2,238 (71.8\%) were men and 877 (28.2\%) were women; 1,352 (43.4\%) patients had no MVD and 1,763 (56.6\%) had MVD. Among patients with MVD, 926 (52.6\%) had two-vessel disease and 837 (47.4\%) had three-vessel disease. Compared with patients with no MVD, patients with MVD were older, had longer pain duration (before the first medical contact), and presented more often with heart failure (Killip class II and III); they were more likely to have diabetes, hypertension, renal dysfunction, and post-procedural flow TIMI $<3$ through the IRA. EF was lower in patients with MVD compared to patients without MVD. Demographic, clinical, laboratory echocardiographic, angiographic, procedural characteristics of analyzed patients and discharge therapy according to the presence of MVD are presented in Table 1.

In $1,075(61 \%)$ patients, revascularization of the remaining stenosis was performed at index hospitalization (median 10.5 days, IQR 7.4-13.5 days), in 602 (34.1\%) patients, revascularization was performed during the second visit (median 1.5 months, IQR 1-2.5 months), while 86 $(4.9 \%)$ patients were treated medically. Among patients in whom revascularization was performed, CABG was performed in $308(18.4 \%)$ patients and PCI (with stent implantation) in 1,368 (81.6\%) patients.

Cardiovascular mortality at 6 years was significantly higher in patients with MVD than in patients with no MVD (10.4\% vs. 4.6\%, p <0.001), as presented in Figure 1.

After adjustment for variables defined in univariate analysis as predictors of cardiovascular mortality, MVD remained an independent predictor of cardiovascular mortality during the 6-year follow-up.

\section{DISCUSSIONS}

The results of the present study confirmed that STEMI patients with MVD had higher long-term mortality compared to STEMI patients with no MVD. After multivariate

TABLE 2. Association between MVD and 6-year cardiovascular mortality (univariate analysis and multivariate Cox regression analysis)

\begin{tabular}{|c|c|c|c|c|}
\hline & HR $(95 \% \mathrm{CI})$ & $\mathrm{p}$ value & HR (95\% CI) & $p$ value \\
\hline Killip II and III at admission & $9.35(6.85-12.76)$ & $<0.001$ & $3.23(3.31-5.36)$ & $<0.001$ \\
\hline $\mathrm{RD}$ & $5.15(3.79-6.99)$ & $<0.001$ & $1.75(1.29-2.49)$ & 0.024 \\
\hline Anterior infarction & $2.18(1.18-2.92)$ & $<0.001$ & & \\
\hline Previous MI & $1.99(1.35-2.93)$ & $<0.001$ & & \\
\hline Age & $1.07(1.06-1.08)$ & $<0.001$ & $1.03(1.02-1.04)$ & $<0.001$ \\
\hline $\mathrm{EF}$ & $0.86(0.84-0.88)$ & $<0.001$ & $0.90(0.70-0.99)$ & $<0.001$ \\
\hline
\end{tabular}


adjustment, MVD remained an independent predictor for long-term mortality (up to six years) in analyzed patients. The percentage of patients with MVD in our study (56.6\%) was similar to the previously published studies and registry analyses in which the percentage of patients with MVD range from $50 \%$ to $65 \%{ }^{1-5}$ The clinical characteristics of our patients with MVD are also in consistence with data found in the literature. ${ }^{1,2,5}$ Patients with MVD are generally an older and sicker cohort (e.g., presenting more frequently diabetes, renal dysfunction, previous coronary disease etc.). ${ }^{1-4}$ This finding can partially explain their worse outcome compared to patients with no MVD. However, we found an independent association between MVD and long-term cardiovascular mortality. We also found a poorer success rate of reperfusion (post-procedural TIMI flow) in patients with MVD, and this is also in consistence with previously published data. ${ }^{3,4,6}$ Our patients with MVD had lower EF than patients without MVD, which could strongly influence survival, but the enzymatic infarct size did not differ between groups. This may suggest that patients with STEMI and MVD had left ventricular dysfunction before the index event as a consequence of their coronary artery disease. ${ }^{2}$

Mortality rates and the independent prognostic impact of MVD on long-term mortality in our study are in consistence with data found in the literature. In a study by Lekston et al., MVD was an independent predictor of 5-year mortality in STEMI patients, predominantly treated with pPCI (around 30\% of patients were treated with thrombolysis, with subsequent early PCI) in this study. ${ }^{2}$ This finding is in consistence with our results and also confirms that the presence of MVD adversely affects long-term prognosis in STEMI patients, regardless of reperfusion strategy. In a study by Park et al. it was found that the presence of MVD was significantly associated with an increased risk for 30-day and 1-year mortality in patients treated with pPCI. ${ }^{5}$ Similar results are reported in studies by De Luca et al. and Sorajja et al.,12 However, there are no data about the prognostic impact of MVD after 1 year in these studies. In a study by de Waha et al., it was reported that the presence of MVD in patients with STEMI treated with contemporary PPCI is independently associated with the occurrence of composite endpoint major adverse cardiac events (MACE) (comprising reinfarction, all-cause death, and newly developed heart failure) at the one-year follow-up. These results could be explained by an important correlation between MVD and increased rates of reinfarction, while the presence of MVD did not predict the all-cause mortality rates of newly developed heart failure. ${ }^{1}$
In more than $60 \%$ of our patients with MVD, revascularization of the remaining stenosis was performed during the index hospitalization. It is well known that preventive PCI to treat non-IRA stenosis early after pPCI reduces the risk of cardiac death, non-fatal reinfarction, or refractory angina by up to $65 \%{ }^{13}$ Despite this, a higher risk for increasing cardiovascular death rates are probably influenced by several factors, and not just by the presence of other significant coronary stenoses. ${ }^{1}$ MVD can be understood as a marker of more advanced CAD and diffuse atherosclerosis, slow flow in critically narrowed non-IRA, and the presence of stunned and hibernating myocardium. That is why the early revascularization of the remaining stenosis, as suggested by current guidelines, is very important. ${ }^{1-4,10,13}$ In acute coronary syndromes, plaque instability may not be limited to the culprit lesion, but may rather involve large areas of the coronary tree because of systemic endothelial dysfunction and higher platelet activity. $3,4,8$ Thus, patients with MVD should benefit of a closer monitoring and follow-up strategy, both during hospitalization and after discharge. This approach should include early and more intense therapeutic measures for classic atherosclerosis risk factors, as well as recurrent ischemia testing, for a longer period of time than just during the first weeks and months after the acute event. ${ }^{1}$

\section{STUDY LIMITATIONS}

This is an observational, prospective, single-center study; however, it included consecutive patients, limiting possible selection bias. The decision whether to revascularize the non-culprit lesions and the timing of revascularization were decided by the interventional cardiologist, who was not part of the study protocol. MVD assessment was performed only by analyzing the invasive coronary angiography, without evaluating the functional significance of the coronary lesions, which could have provided additional information (e.g., fractional flow reserve). Nevertheless, most clinical trials on STEMI in MVD patients are based on angiographic judgment only. Primary PCI was predominantly performed using bare metal stents. Patients were treated with clopidogrel; there were no patients treated with more recently developed antithrombotic drugs (since ticagrelor was not available for routine administration to patients at the time of their entry into the Registry), and this could have influenced the prognosis of the patients, i.e. reduce the occurrence of cardiovascular deaths. We excluded patients presenting with cardiogenic shock; by definition, these patients fall into the highest risk category, and their treatment differs from the overall pPCI population.10 
Also, the protocol of the study stipulated that patients with cardiogenic shock at admission should have separate risk stratification and a different treatment strategy.11 In this study we did not analyze whether the relationship between MVD and mortality would be different in case of other treatment strategies.

\section{CONCLUSIONS}

In STEMI patients treated with pPCI, the presence of MVD is an independent predictor for higher long-term cardiovascular mortality, despite early revascularization of the remaining stenosis in non-IRA.

\section{CONFLICT OF INTEREST}

Nothing to disclose.

\section{REFERENCES}

1. De Waha S, Eitel I, Desch S, et al. Impact of multivessel coronary artery disease on reperfusion success in patients with ST elevation myocardial infarction: A substudy of the AIDA STEMI trial. Eur Heart J Acute Cardiovascular Care. 2018;7:28-37. doi: 10.1177/2048872615624240.

2. Lekston A, Tajstra M, Gąsior, et al. Impact of multivessel coronary disease on one-year mortality in patients with STelevation myocardial infarction undergoing percutaneous coronary intervention. Kardiol Pol. 2011;69:336-343.

3. Batra MK, Rasool SI, Solangi BA, et al. Multivessel disease as a prognostic marker in patients presenting for primary percutaneous coronary intervention. J Ayub Med Coll Abbottabad. 2018;30:534-538.

4. De Luca G, Gibson M, Cutlip D, et al. Impact of multivessel disease on myocardial perfusion and survival among patients undergoing primary percutaneous coronary intervention with glycoprotein IIb/IIIa inhibitors. Arch Cardiovasc Dis. 2013;106:155-161. doi: 10.1016/j.acvd.2012.12.007.
5. Park DW, Clare RM, Schulte PJ, et al. Extent, location, and clinical significance of non-infarct-related coronary artery disease among patients with ST-elevation myocardial infarction. JAMA. 2014;312:2019-2027. doi: 10.1001/ jama.2014.15095.

6. Tarantini G, Napodano M, Gasparetto N, et al. Impact of multivessel coronary artery disease on early ischemic injury, late clinical outcome, and remodeling in patients with acute myocardial infarction treated by primary coronary angioplasty. Coron Artery Dis. 2010;21:78-86. doi: 10.1097/ MCA.obo13e328335a074.

7. De Luca G, Parodi G, Sciagrà R, et al. Impact of multivessel disease on infarct size among STEMI patients undergoing primary angioplasty. Atherosclerosis. 2014;234:244-248. doi: https://doi.org/10.1016/j.atherosclerosis.2014.02.031.

8. Widimsky P, Holmes DR Jr. How to treat patients with STelevation acute myocardial infarction and multi-vessel disease? Eur Heart J. 2011;32:396-403. doi: 10.1093/eurheartj/ ehq410.

9. Greshlick AH, Khan JN, Kelly DJ, et al. Randomized trial of complete versus lesion-only revascularization in patients undergoing primary percutaneous coronary intervention for STEMI and multivessel disease. J Am Coll Cardiol. 2015;65:963972. doi: 10.1016/j.jacc.2014.12.038.

10. Neumann FJ, Sousa-Uva M, Ahlsson A, et al. 2018 ESC/EACTS Guidelines on myocardial revascularization The Task Force on myocardial revascularization of the European Society of Cardiology (ESC) and European Association for CardioThoracic Surgery (EACTS). Eur Heart J. 2019;40:87-165. doi: https://doi.org/10.1093/eurheartj/ehy394.

11. Mrdovic I, Savic L, Lasica R, et al. Efficacy and safety of tirofiban-supported primary percutaneous coronary intervention in patients pretreated with $600 \mathrm{mg}$ clopidogrel: results of propensity analysis using the Clinical Center of Serbia STEMI Register. Eur Heart J Acute Cardiovascular Care. 2014;3:56-66. doi: 10.1177/2048872613514013.

12. Sorajja P, Gersh BJ, Cox DA, et al. Impact of multivessel disease on reperfusion success and clinical outcomes in patients undergoing primary percutaneous coronary intervention for acute myocardial infarction. Eur Heart J. 2007;28:1709-1716. doi: 10.1093/eurheartj/ehm184.

13. Wald DS, Morris JK, Wald NJ, et al. Randomized trial of preventive angioplasty in myocardial infarction. N Engl J Med. 2013;369:1115-1123. doi: 10.1056/NEJMoa1305520. 